$\underline{\text { Preprint typeset in JHEP style - HYPER VERSION }}$

LU-TP $05-25$,

LUNFD6/(NFFL-7223)2005

hep-ph/0505181

\title{
QCD-supression by Black Hole Production at the LHC
}

\author{
Leif Lönnblad and Malin Sjödahl \\ Dept. of Theoretical Physics, Sölvegatan 14A, S-223 62 Lund, Sweden \\ E-mail: Eeif.Lonnblad@thep.lu.se and Malin.Sjodahl@thep.lu.se \\ Torsten Åkesson \\ Dept. of Physics, Lund University, Box 118, S-221 00 Lund, Sweden \\ E-mail: Torsten.Akesson@hep.lu.se
}

\begin{abstract}
Possible consequences of the production of small black holes at the LHC for different scenarios with large extra dimensions are investigated. The effects from black hole production on some standard jet observables are examined, concentrating on the reduction of the QCD cross section. It is found that black hole production of partons interacting on a short enough distance indeed seem to generate a drastic drop in the QCD cross section. However from an experimental point of view this will in most cases be camouflaged by energetic radiation from the black holes.
\end{abstract}




\section{Contents}

1. Models of large extra dimensions 2

1.1 Basics of ADD 2

1.2 Basics of RS 1

2. Black hole production and decay

2.1 CHARYBDIS 5

3. Uncertainties 6

3.1 Non black hole gravitational events

3.2 The formation of a black hole

3.3 The decay of a black hole

4. Results 8

5. Conclusion and outlook 12

Since the theory of Large Extra Dimensions by Arkani-Hamed Dvali and Dimopoulos (ADD) first saw light in 1998 [1] there has been much discussion in the literature about the prospect for observing these large extra dimensions in colliders (see e.g. [2-6]). In particular the signal of small black holes which can be produced if the Planck scale, $M_{\mathrm{P}}$, is of order $\mathrm{TeV}$ has been discussed. However, there is another characteristic of black hole events; the signal of no signal [7] . If we e.g. consider the dijet cross section as a function of invariant mass, $\sqrt{\hat{s}}$, then for some large invariant mass, the partons which otherwise would have undergone a QCD scattering may instead be trapped in a black hole which would lead to a falling dijet cross section as a function of invariant mass.

This argument is clearly oversimplified. Requiring a large $\hat{s}$ ensures a small extension of the interaction in beam direction. However, to form a black hole the energy has to be well confined also in transverse direction. The absolute range of a QCD interaction is set by the virtuality of the propagator, $Q^{2}$. This sets the timescale of the fluctuation and we therefore expect that interactions with a large enough virtuality takes place over a small enough space such that it is really possible to form a black hole, at least if the mass is high enough to avoid uncertainties due to quantum gravity. Hence, the dijet cross section would only fall off for high enough $E_{\perp}$ of the jets. Of course, some of the produced black holes may themselves decay into hard partons, filling up the jet spectrum at high masses and $E_{\perp}$, but we expect that spectra to look quite different from standard QCD.

For energies much larger than the Planck scale, corresponding to a Compton wavelength much smaller than the Schwarzschild radius of a black hole with that energy, we 
expect classical gravity to give the correct result, and a produced black hole would evaporate by emitting particles with the thermal spectra from Hawking radiation. The problem is that in colliders we would first probe the Planck scale region where quantum gravitational effects should come into play.

Approaching the Planck scale from below, where gravity starts to become important, but still is not strong enough to trap partons in black holes, we would expect gravitational scattering, to leading order simply $2 \rightarrow 2$ partonic scatterings [8-10]. As the energy increases, the gravitational scattering would transform into small black hole events. On the other hand approaching from above using the approximation of Hawking radiation we would expect the black holes to decay to a few particles.

In this transition region we are sensitive to unknown quantum gravity effects, but from general continuity arguments we would expect the black holes to decay into a small number of particles, preferably two. Then as the energy increases we expect the black holes to emit more and more particles looking more and classical and, in the end for $M \gg M_{\mathrm{P}}$, we would expect the thermal spectra from Hawking radiation.

Hence, in searching for a drop in the cross section, a more complicated jet spectra will appear with increasing $E_{T}$ scales. First there will be an increase of the cross section due to gravitational interactions. Then, as black holes begin to form, they are expected to decay to relatively few particles which will sometimes be identified as jets, resulting in an increase of the cross section. At high enough jet scales, however, we expect the jet cross section to completely die out, since the QCD component of the hard scattering will disappear and the black hole decay products will be softer as the black hole masses increase.

From the point of view of searching for large extra dimensions it is, of course, the increased cross section which will be easiest to see, already at scales much below where black holes are formed. But an increase in the jet cross section could be attributed to many different kinds of new physics, while the disappearance of Standard Model cross sections is not normally expected from new physics processes.

So, while the above mentioned effects (gravitational scattering and black hole decay products) are well worth looking for, we will devote this paper to the black hole 'eating' of the QCD cross section, and we will do this by using phenomenological event generator models. We intend to get back to the other issues in future publications.

I this paper we start with a brief review of the ADD and the, somewhat different, Randall-Sundrum (RS) [11] extra-dimension scenarios in section 1. The model for generating and decaying black holes is described in section 2, while the many theoretical uncertainties are discussed in section 3. In section t we present and comment on our results and in section 5 we make the concluding remarks and suggest further studies.

\section{Models of large extra dimensions}

\subsection{Basics of ADD}

The aim of the Arkani-Hamed Dvali and Dimopoulos (ADD) model [1] is to solve the hierarchy problem of the differences in scale between the electro-weak scale, $100-1000$ $\mathrm{GeV}$, and the scale where gravitation becomes important, $10^{19} \mathrm{GeV}$. This is done by 
introducing large extra dimensions with some compactification radius, $R$. Although the compactification radius is typically taken to be the same in all extra dimensions it could in principle vary. For distances much smaller than the size of the extra dimensions (here assumed to be the same) we will then have a Newton's law of the form [1]

$$
V(r) \sim \frac{M}{M_{\mathrm{P}}^{n+2}} \frac{1}{r^{n+1}}
$$

where $n$ is the number of extra dimensions and $M_{\mathrm{P}}$ the fundamental $n+4$ dimensional Planck mass. On the hand, for $r \gg R$ gravity has expanded to the full volume of the extra dimensions and we get [1]

$$
V(r) \sim \frac{M}{M_{\mathrm{P}}^{n+2}} \frac{1}{R^{n}} \frac{1}{r}
$$

But this must equal to Newton's law in $3+1$ dimensions so we conclude that the observed four dimensional Planck mass $M_{\mathrm{P} 4}$ is (up to small volume factors)

$$
M_{\mathrm{P} 4}^{2} \sim M_{\mathrm{P}}^{n+2} R^{n}
$$

This explains how we could have a fundamental Planck scale almost at the electroweak scale but an observed Planck scale at $10^{19} \mathrm{GeV}$. Requiring that the fundamental Planck scale is the same as the electroweak scale, $\sim \mathrm{TeV}$, gives a compactification radius of the solar system for one extra dimension. This is trivially excluded from observations. Two extra dimensions gives $R \sim \mathrm{mm}$, which is indirectly excluded from various cosmological and astrophysical constraints. The same holds for $R \sim \mathrm{nm}$, corresponding to three extra dimensions. However for four or more extra dimensions it is still possible to have a fundamental Planck scale at the order of a TeV (A more complete listing is given in e.g. [6]).

Since the black holes considered here are well within the range $r \ll R$, the Schwarzschild radius can be calculated analogous to the $3+1$ dimensional case. The result is [12]

$$
r_{\mathrm{Sch}}=\frac{1}{\sqrt{\pi} M_{\mathrm{P}}}\left[\frac{M_{\mathrm{BH}}}{M_{\mathrm{P}}} \frac{8 \Gamma\left(\frac{n+3}{2}\right)}{n+2}\right]^{\frac{1}{n+1}}
$$

where $\Gamma$ is the Euler gamma function.

The temperature is given by [12]

$$
T=\frac{n+1}{4 \pi r_{\mathrm{Sch}}}
$$

This means that more massive holes are colder. The mass dependence is however much weaker than in 4 dimensions since the radius changes less with mass. The dependence on the number of extra dimensions is dominated by the factor $n+1$ in the numerator. Hence black holes in many dimensions are hotter. 


\subsection{Basics of RS}

A somewhat different model was introduced one year after the ADD-model by Randall and Sundrum [11]. In the Randall-Sundrum model, RS, which has only one extra dimension, the metric doesn't factorize. Instead the 4 dimensional metric is multiplied by a "warp" factor which depends on the coordinate, $y \in[-\pi, \pi]$, in the fifth dimension.

$$
d s^{2}=e^{-2|y| / l} \eta_{i j} d x^{i} d x^{j}+d y^{2}
$$

In the above expression $\eta_{i j}$ is our normal (flat) four dimensional metric with indices running from 0 to 3 and $l$ is the radius in the anti-de Sitter space. By taking the warp factor to be an exponent, and by assuming the gauge fields (and hence us) to live in the most shrinked slice, $y=\pi$, of the five dimensional world, a large hierarchy can be accomplished by letting gravity propagate in the full space.

We note that it has been suggested (see eg. [13]) that black holes in the RS model can only be formed on the Planck brane, which effectively means that there would be no black holes produced in a collider. However, it was also suggested [14] that the naked singularity obtained instead, could be covered on the Standard-Model brane. Such a singularity could then be produced in a collider and would evaporate as gravitational radiation into the bulk (where it is naked) at the Planck time scale. As this radiation is not detected it would appear as missing energy.

On the other hand, if a black hole is formed, but is stable on collider time scales as argued in [15], the hole could (if gauge charged) be detected. It is, however, unlikely that this would affect the signals studied here, and we have chosen to ignore this effect.

If a RS black hole is sufficiently small, such that it is not affected by the bulk curvature, the radius is still given by eq. (1.4) [16]. Hence, in either of the above mentioned cases for black holes/naked singularities in the Randall-Sundrum scenario, there will be no Hawking radiation which populates the spectra, but there will be a drop in total cross section for standard model interactions. Furthermore the cross section would be the same as in the ADD scenario with one extra dimension for small enough black holes [16].

For the purpose of this paper, we ignore all the uncertainties of whether or not black holes are possible in the RS model. In fact, we do not use any details of the RS model, but use it only as an example of a model which could produce black holes/naked singularities at large cross sections at a collider, but where the decay products of the hole would not be detectable. For our investigations this is then a best-case scenario.

\section{Black hole production and decay}

To study the effect on QCD-jets on LHC we have used PYTHIA [17] to generate the QCD events. To lowest order we here have two partons with energy fractions, $x_{1}$ and $x_{2}$, and an invariant mass, $\hat{s}=x_{1} x_{2} s$, which scatter and give rise to two outgoing partons with

transverse momenta, $p_{\perp} \approx \sqrt{Q^{2}}$. For this process to be trapped in a black hole, we require the whole process to be located within the Schwarzschild radius, $r_{\mathrm{Sch}}(\hat{s})$. Looking at the 
momenta of the incoming partons in their combined rest frame we must require that their wavelength, $\lambda_{l} \propto 2 / \sqrt{\hat{s}}$, is less than $r_{\mathrm{Sch}}$. The corresponding requirement in the transverse direction gives the requirement: $\lambda_{\perp} \propto 1 / p_{\perp}<r_{\mathrm{Sch}}$.

Of course, it is questionable if these requirements are enough, maybe the wavelengths should be much smaller than $r_{\mathrm{Sch}}$. In any case it is reasonable to introduce a parameter, and we will require $\lambda<r_{\mathrm{Sch}} / P$, where we use $P=1$ as a standard value. From the longitudinal requirement we then get a minimum mass of a black hole from

$$
M_{\min }=2 P / r_{\mathrm{Sch}}\left(M_{\min }\right) .
$$

We then get a cutoff in the standard QCD cross section, given by the following stepfunctions (which in general could be replaced by more smooth suppression functions):

$$
\begin{aligned}
\frac{d \sigma_{\mathrm{QCD}}\left(Q^{2}\right)}{d Q^{2} d \hat{s}}=\int & d x_{1} d x_{2} \sum_{i, j} f_{i}\left(x_{1}, Q^{2}\right) f_{j}\left(x_{2}, Q^{2}\right) \frac{\hat{\sigma}_{i j}^{\mathrm{QCD}}\left(\hat{s}, Q^{2}\right)}{d Q^{2}} \times \\
& \delta\left(\hat{s}-x_{1} x_{2} s\right)\left[1-\Theta\left(\hat{s}-M_{\mathrm{min}}^{2}\right) \Theta\left(Q^{2}-\frac{P^{2}}{r_{\mathrm{Sch}}^{2}(\hat{s})}\right)\right] .
\end{aligned}
$$

where the sum runs over all parton types.

Instead of the QCD process we will get black holes with the cross section

$$
\frac{\sigma^{\mathrm{BH}}(\hat{s})}{d \hat{s}}=\int d x_{1} d x_{2} \sum_{i, j} f_{i}\left(x_{1}, Q^{2}\right) f_{j}\left(x_{2}, Q^{2}\right) \hat{\sigma}^{\mathrm{BH}}(\hat{s}) \delta\left(\hat{s}-x_{1} x_{2} s\right) \Theta\left(\hat{s}-M_{\min }^{2}\right),
$$

where $Q=P / r_{\text {Sch }}(\hat{s})$. The partonic cross section is simply given by $\hat{\sigma}=\pi r_{\text {Sch }}^{2}$, but also here one could imagine a smooth transition and a factor in front (see section 3.2 below).

\subsection{CHARYBDIS}

We here describe the most important properties of the black hole event generator CHARYBDIS, which we have used to generate black holes and their decays. The complete references are $[18,19]$.

In CHARYBDIS two partons create a black hole according to $(2.3)^{1}$, which then decays by Hawking radiation. The procedure of the decay is such that momentum, charge, color and baryon numbers are conserved. The decay products are chosen democratically among the standard model particles according to their degrees of freedom (color and spin). The energy of an emitted particle is taken from a Planck spectra modified with the gray-body factor of the particle. The temperature is given by eq. (1.5) where the black hole mass by default is taken to be the remaining mass of the hole, but this may be modified such that the initial temperature is kept throughout the evaporation. The motivation for keeping the initial temperature could be that is is anyhow dubious to treat the black hole as a thermalized object considering the speed of the decay [4].

\footnotetext{
${ }^{1}$ By default the scale $Q^{2}$ is taken to be $\hat{s}$ in CHARYBDis, but we have used the option with $Q^{2}=1 / r_{\text {Sch }}^{2}[5]$, since this gives a more continuous transition from eq. (2.2).
} 
One problem is that even if a black hole would be created well above the Planck scale, i.e. well within the classical regime, it would evaporate and eventually reach the Planck scale. And, although we do not know what physics looks like at the Planck scale, there must be a way of terminating the decay.

The CHARYBDIS method for this is to have a free parameter, set by the user, which gives the number of particles the hole decays to in the end, where the end can be defined either as when the black hole reaches the Planck mass, or - if it happens before - when one of the emitted particles happens to get a momentum so large that energy and momentum conservation would be violated for a two body decay.

\section{Uncertainties}

First of all it should be pointed out that the major lack in our description is the absence of a theory for quantum gravity. As we do not know what physics we will encounter at the Planck scale it is impossible to make an error estimate in the traditional sense. However we may still discuss which uncertainties will effect our results. Roughly these can be classified in three groups. The first concerns non black hole gravitational events, the second the production of black holes, and the third deals with the decay of the black holes.

Apart from the uncertainties associated with quantum gravity there is of course also an uncertainty in the QCD jet cross section. A recent evaluation of the effect from the uncertainties in the parton distribution functions, and in the NLO QCD theory, is published in [20]. In this paper a comparison is made between the calculated cross section and the Tevatron Run 1b data, and the uncertainty in the jet cross section for the LHC is estimated to be up to a factor 2.5 at a jet-ET of $5 \mathrm{TeV}$. This effect is important for many QCD and other Standard Model studies, however, it is an effect that can be neglected for the main conclusions in this paper as can be seen in e.g. figure 3 .

\subsection{Non black hole gravitational events}

As mentioned in the introduction it is highly unlikely that black holes is the major gravitational effect. In the ADD scenario the amplitude for perturbative scattering is calculated in [8] and the dijet cross section is spelled out (using the Planck scale as cut of for the Kaluza Klein modes) in e.g. [9]. While the effect on jet production from gravitational scattering is large, it has the disadvantage of depending on a free parameter for the cutoff. Furthermore, as it is a perturbative expansion it cannot be applied close in the Planck region. This means that when searching for the signal of a disappearing QCD cross section, we have to do that in a spectra which is already modified by gravitational scattering in a basically unknown way. In this paper we will not further investigate this issue, and these processes are not included in the simulation. Although they should have a large effect

on the cross section close to the Planck scale, we will here mostly be concerned with the behavior well beyond this scale. 


\subsection{The formation of a black hole}

There are several unresolved issues concerning the formation of mini black holes. Firstly there is an uncertainty already at the classical level. The naive formula is $\sigma\left(M_{\mathrm{BH}}\right)=\pi r_{\text {Sch }}^{2}$. Classical numerical simulations indicates that this value should be multiplied by a factor $\sim 0.7-3$, increasing with the number of extra dimensions [21,22]. (For a discussion about the effects of quantum fluctuations based on wave packages, see [23,24].) During the formation some energy may be lost as gravitational energy reducing the remaining mass for Hawking radiation $[22,25]$.

Secondly the factorization of the parton level cross section can be questioned since the process is inherently non-perturbative [26].

Another fundamental issue concerns the onset of black hole production. One may argue that no black holes should be formed below roughly the Planck scale since this is (approximately) the mass scale where the Compton wave length equals the black hole radius. This is the view point taken in eq. (2.1). Combining eq. (2.1) and eq. (1.4) we get the following relation for $M_{\min }$

$$
M_{\min }=M_{\mathrm{P}} \pi^{\frac{n+1}{2(n+2)}}\left[\frac{n+2}{8 \Gamma\left(\frac{n+3}{2}\right)}\right]^{\frac{1}{n+2}}(2 P)^{(n+1) /(n+2)}
$$

We have used $P=1$ as a standard value in this paper. Numerically the value of $M_{\min }$ is then approximately twice the Planck mass.

As for $Q^{2}$, used in the parton distribution functions when generating the black holes, we like to use the same constant in the inverse relation between length and mass as in eq. (2.1) and this is what gave us $Q=P / r_{\mathrm{Sch}}$. While, in principle, the scale in the parton distribution functions and the minimal mass could be varied independently we have chosen not to.

One could also think of more sophisticated ways, where the nature of the force is taken into account, of relating the 'interaction distance' to momentum but that is beyond the scope of this article.

\subsection{The decay of a black hole}

It is common to divide the decay of a mini black hole into 3 different phases. In the first the asymmetry of the black hole, due to energy momentum and gauge charge distribution, is lost. This is therefore referred to as the "balding phase", and it cannot be incorporated into CHARYBDIS as the spectra of this phase is not known. Furthermore, gravitational radiation can be emitted on the brane. As this radiation is not detected it will appear as a missing energy. This radiation has also not been included in CHARYBDIS, where black holes decay only into standard model particles.

The second phase is the Hawking evaporation phase. This is the phase most accurately described by CHARYBDIS. However there are still a few discrepancies. One is that CHARYBDIS uses the spectra of a non-rotating Schwarzschild black hole, while the differential cross section would favor a Kerr hole with large angular momentum, see e.g. [5]. A 
rotating black hole may emit a significant part of the total radiation into the bulk [27-30]. There is not yet a consensus in the literature about how much is emitted into the bulk, but it is not inconceivable that it is as much as 50\%. As large impact parameters are favored this may significantly diminish the observed radiation. Also, it may be possible for the black hole to recoil off the brane, emitting further Hawking radiation in the bulk [31,32].

In principle the gauge charges of the black holes should also be taken into account. In the case of no extra dimensions and larger black holes $\left(M_{\mathrm{BH}} \gg M_{\mathrm{P}}\right)$ the suppression of charged particles due to the electrostatic potential is argued to be a small effect of a few per cent [33]. But since QCD is a significantly stronger force, and since the black holes considered here are small, the effect of QCD may be sizable. Again this is not properly included in CHARYBDIS. There is, however, effectively a bias for events with few charges since charge is conserved, implying that more events with large charge emission are thrown away.

It can also be questioned if it is correct to treat these mini black holes as thermalized considering their rapid decay $[4]^{2}$. From the point of view of this study a varying temperature is however the most conservative choice since a hotter (thermalized) hole gives fewer decay products and hence is more difficult to distinguish from the QCD dijet background. We hence chose a time varying temperature. An example of the difference between a varying and a non varying temperature can be found in [26].

Furthermore, it has been argued that the rapid decay may lead to the black hole becoming surrounded by a chromosphere of soft partons which could suppress emission of hard partons [34]. Such effects are not included in CHARYBDIS and, again, our results will be on the conservative side w.r.t. the disappearance of hard jets.

In the final phase the black hole will disappear. Even if a black hole is produced well above the Planck scale it will evaporate and eventually enter the Planck region. As previously mentioned, the CHARYBDIS treatment of this problem is to let the user chose the number of particles to which the black hole should decay in the end. For this study we have consistently used 2, partly because this gives the most continuous transition from a non black hole gravitational event, and partly since it is the most conservative assumption as it gives the largest number of very energetic particles. For the same reasons we have chosen to define the "end" of the evaporation as whatever happens first of the black hole reaching the Planck mass, or a decay product having a forbidden momentum as explained in section 2.1.

In total, the "mistreatment" by effectively using (Schwarzschild black hole) Hawking radiation for all phases, terminated by a two-particle decay, will lead to a maximum radiation of standard model particles and to a harder spectrum than if additional effects are taken into account. The simulation is therefore based on conservative assumptions wrt. searching for the disappearance of standard QCD jet production.

\section{Results}

To investigate possible effects of black-hole production on standard QCD observables, we

\footnotetext{
${ }^{2}$ On the other hand the decay may be slowed down as argued in [15].
} 
(a)

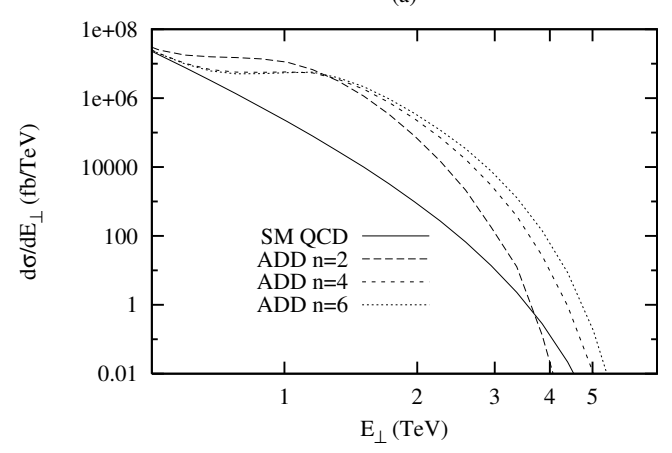

(b)

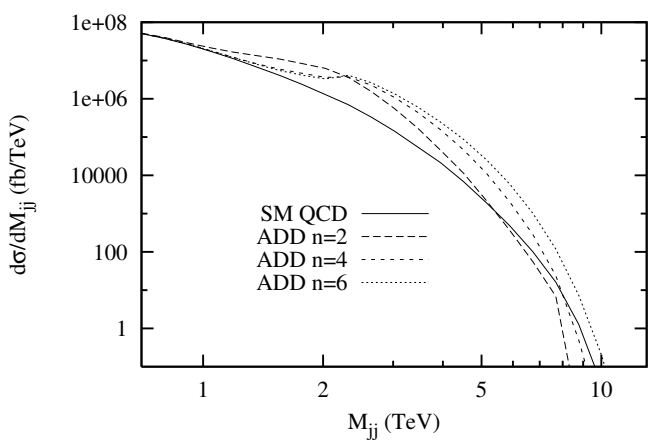

Figure 1: Differential jet cross sections for the ADD scenario with $M_{\mathrm{P}}=1 \mathrm{TeV}$ and $P=1$ for two (long-dashed line), four (short-dashed) and six (dotted) extra dimensions, compared with the Standard Model prediction (full line). (a) shows the cross section as a function of the transverse energy of the hardest jet and (b) the cross section as a function of the invariant mass of the two hardest jets in an event.

have used the PYTHIA event generator (version 6.227) [17] for the standard QCD processes together with the CHARYBDIS program [18] for the production of black holes and their decays. We have studied predictions for the $E_{\perp}$ spectrum of jets and the dijet invariant mass spectrum at the LHC using a cone algorithm with a cone radius of 0.7 and a minimum $E_{\perp}$ of $250 \mathrm{GeV}$ assuming a calorimeter with $0.1 \times 0.1$ resolution in the pseudo rapidity interval $|\eta|<2.5$.

PYTHIA was set to generate standard QCD events using CTEQ5L parton distributions [35], but for the extra dimensions scenarios, the differential cross section was cutoff according to eq. (2.2).

Black holes were produced according to eq. (2.3) and decayed with the CHARYBDIS program, while additional parton showering was handled by PүтніА. ${ }^{3}$ Apart from the the scale in the parton distribution functions, the varying Planck mass, minimal mass, number of extra dimensions and the decision of when to terminate the decay, the default settings of CHARYBDIS were used.

In figure 1 the differential jet cross section is shown as a function of the transverse energy of the hardest jet, and the dijet cross section as a function of dijet invariant mass for the two hardest jets, for two, four and six extra dimensions (ADD), $M_{\mathrm{P}}=1 \mathrm{TeV}$ and $P=1$ (corresponding to $M_{\min }=2.24 \mathrm{TeV}$ ). Studying the case of two extra dimensions we find that, although the main effect is an increase in cross section due to the production of black holes in the regions just above the Planck mass, the spectra do indeed fall below the standard model spectra for very large $E_{\perp}$ and invariant masses. The effect should be clearly visible at the LHC where we would e.g. expect over a hundred events with $M_{j j}$ above $8.5 \mathrm{TeV}$ with an integrated luminosity of $100 \mathrm{fb}^{-1}$ (corresponding to one year of running at $10^{34} \mathrm{~cm}^{-2} \mathrm{~s}^{-1}$ ) for standard QCD, while with black hole production there would be basically none. In the $E_{\perp}$ spectrum the effect would be harder to see, and only a

\footnotetext{
${ }^{3}$ Hadronization was not included in the simulations presented here, but we have checked that our results do not change if hadronization is added.
} 

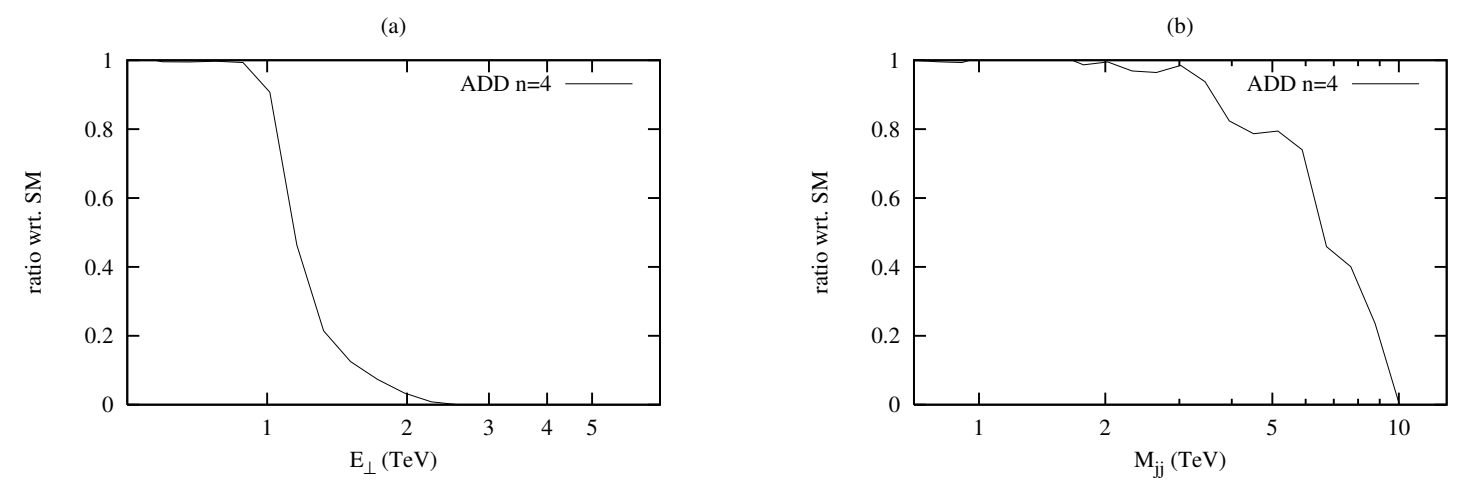

Figure 2: The number of pure QCD events for 4 extra dimensions, $M_{\mathrm{P}}=1$ and $P=1$ divided by the number of QCD events in a Standard Model world as a function of (a) the transverse energy of the hardest jet and (b) the invariant mass of the two hardest jets in an event.

couple events expected above $4 \mathrm{TeV}$ from standard QCD would disappear in a $n=2 \mathrm{ADD}$ scenario.

However, two extra dimensions is excluded from observations, and if we increase the number of extra dimensions the temperature rises according to eq. (1.5). As seen in figure — this results in a drastic increase in the number of hard jets, making it seem rather unlikely that we will observe the QCD drop at LHC without further efforts to distinguish QCD and black hole jets. But in principle it is an enormous effect. This can be illustrated by plotting the ratio of QCD events in an ADD world, with e.g. 4 extra dimensions, and QCD events in a four dimensional standard model world. This is shown in figure 2.

The QCD drop can also be clarified by decomposing the contribution to the cross section of standard events and black hole decay products as in figure 3 . As expected the 'shoulder' in the spectra is completely dominated by the black hole decay products. The drop in the QCD cross section is also seen, however, from an experimental point of view it is completely hidden behind the black hole decay products.

Eventually, for high enough energies the QCD drop must appear, since light black holes cannot decay to particles with energy greater than half the black hole mass, and heavier black holes typically will produce less energetic decay products as the temperature is lower.

However, as seen in figure 4 for a imagined $40 \mathrm{TeV} p p$-collider it turns out that the (large) probability for producing a heavy black hole, which then emits an unlikely heavy (Boltzmann suppressed) particle, dominates over the QCD cross section for rather large transverse energies and invariant dijet masses.

The effect of varying $P$, and hence also the minimal mass $M_{\min }$, is shown in figure 5 where $P=1$ for $M_{\mathrm{P}}=1 \mathrm{TeV}$ (giving $M_{\min }=2.24 \mathrm{TeV}$ ) is compared to $P=1 / 2$ for $M_{\mathrm{P}}=2 \mathrm{TeV}\left(M_{\min }=2.51 \mathrm{TeV}\right)$ and $P=1$ for $M_{\mathrm{P}}=2 \mathrm{TeV}\left(M_{\min }=4.48 \mathrm{TeV}\right)$. Comparing the lines which have the same $M_{\mathrm{P}}$ but differ in $P$ and $M_{\min }$ we see that the extra black holes which are produced if $M_{\min }$ is lowered, contribute to the low energy end of the spectrum despite the fact that they are hotter. This is because the emitted quantum must have an energy less than half the black hole energy. On the other hand, the high 
(a)

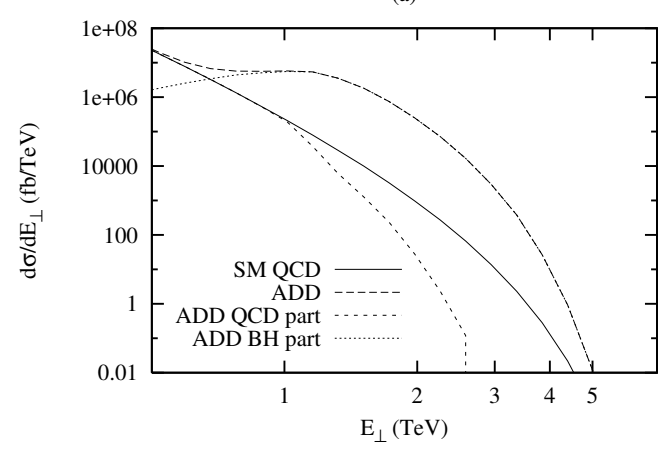

(b)

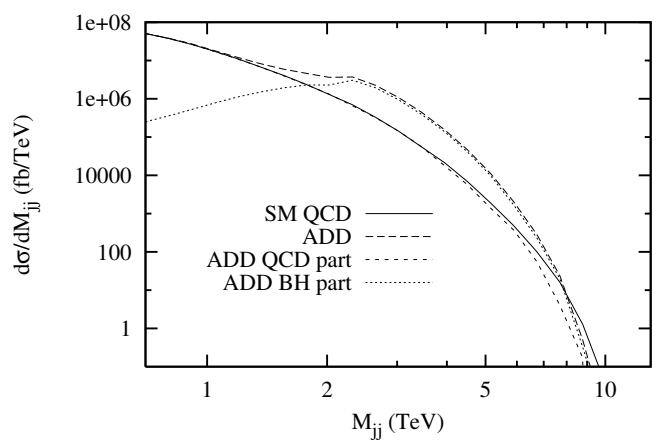

Figure 3: Differential jet cross sections for the ADD scenario with $M_{\mathrm{P}}=1 \mathrm{TeV}$ and $P=1$ for four extra dimensions (long-dashed line), compared with the Standard Model prediction (full line). Also shown are the contributions from events with (dotted line) and without (short-dashed line) black holes. (a) shows the cross section as a function of the transverse energy of the hardest jet and (b) the cross section as a function of the invariant mass of the two hardest jets in an event.

(a)

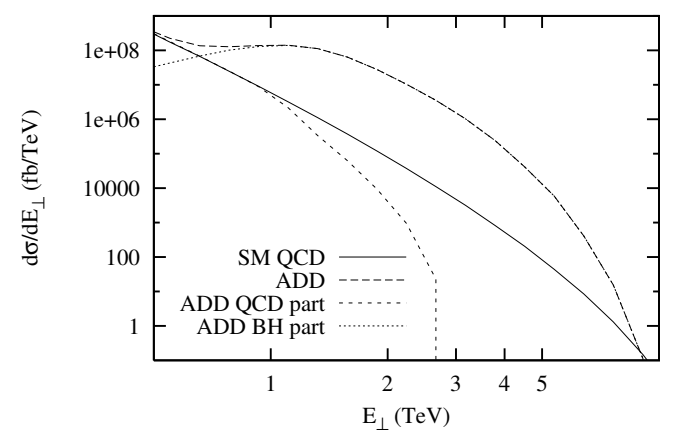

(b)

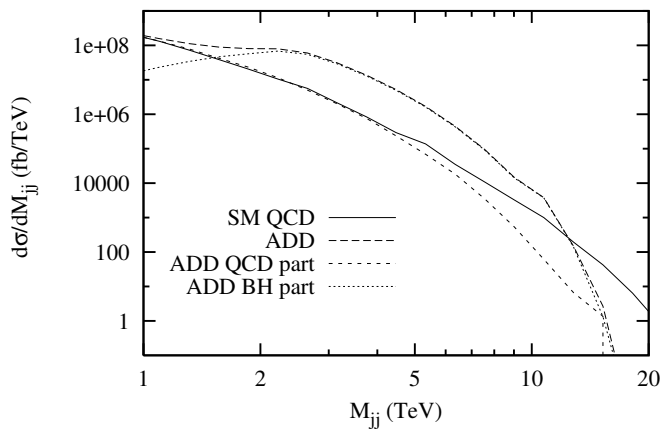

Figure 4: Differential jet cross sections at a $40 \mathrm{TeV}$ pp-collider for the ADD scenario with $M_{\mathrm{P}}=1 \mathrm{TeV}$ and $P=1$ for four extra dimensions (long-dashed line), compared with the Standard Model prediction (full line). Also shown are the contributions from events with (dotted line) and without (short-dashed line) black holes. (a) shows the cross section as a function of the transverse energy of the hardest jet and (b) the cross section as a function of the invariant mass of the two hardest jets in an event.

energy end of the spectra, where the total cross section eventually would fall below the standard model QCD cross section, is left more or less unaffected. This means that the choice of $M_{\min }$ is not particularly important for determining the point where the cross section drop would be observed. Unfortunately this does not imply that it is insensitive to Planck scale physics as recoil effects on the black hole emitting the energetic quanta cannot be neglected.

An increased Planck mass results in a later onset of black hole production and therfore less particles in the low energy end of the black hole decay spectrum. On the other hand there are more particles in the high energy end as the temperature is increased according to eq. (1.5). For a higher Planck mass it would therfore, as expected, be more difficult to observe the QCD drop. 
(a)

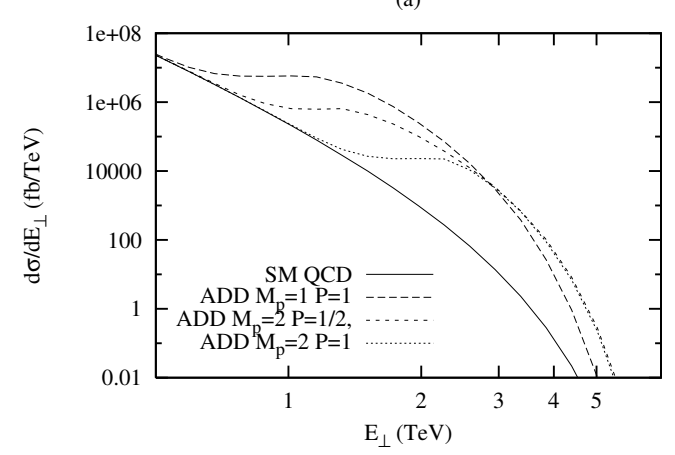

(b)

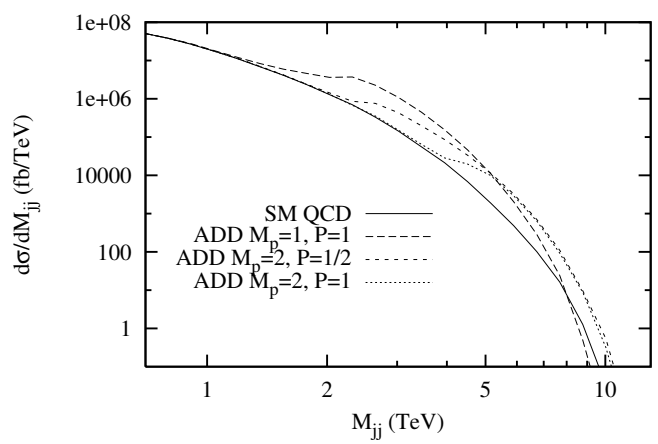

Figure 5: Differential jet cross sections for the ADD scenario for four extra dimensions with $M_{\mathrm{P}}=1 \mathrm{TeV}$ and $P=1$ (long-dashed line), $M_{\mathrm{P}}=2 \mathrm{TeV}$ and $P=1$ (short-dashed) and $M_{\mathrm{P}}=2 \mathrm{TeV}$ and $P=1 / 2$ (dotted line) compared with the Standard Model prediction (full line). (a) shows the cross section as a function of the transverse energy of the hardest jet and (b) the cross section as a function of the invariant mass of the two hardest jets in an event.

One could try to eliminate the extremely energetic black hole events with a thrust cut to regain the QCD drop, but it does not work particularly well since the rest of the black hole which emitted the energetic quanta will have a large momentum in opposite direction. The event will thus (from a clustering point of view) look like a dijet event. Another option is to try a smaller cone radius. This has a significant effect in the case of 2 extra dimensions, making the drop clearly visible also in the $E_{\perp}$-spectrum, but turns out to be less effective in the higher dimensional cases since the black holes there are hotter.

The overall impression for the ADD scenario is thus that it will be hard to observe the QCD drop without further efforts to discriminate between the QCD and black hole radiation. On the other hand we have made the case worse than it may be in several ways. We have ignored that some energy will be carried away by invisible gravitational radiation, thus reducing the observed background from black hole decay products, and we have maximized the number of energetic particles by choosing a varying temperature and a 2-body decay in the end of the evaporation.

If the black holes do not decay on collider timescales, or if a naked singularity which decays in the bulk is formed, as may be the case in the Randall-Sundrum scenario, there is no radiation to camouflage the QCD cross section disappearance. Its disappearance may then be a key signal, as shown in figure 6 .

\section{Conclusion and outlook}

We have seen that black hole production of partons interacting on a short enough distance indeed seem to generate a drastic drop in the QCD cross section at LHC. However if the created black holes decay on collider timescales as expected in the ADD scenario, this drop will (naively) be completely hidden by the black hole decay products even for rather large transverse energy/invariant dijet mass. On the other hand, if the black holes are stable on 
(a)

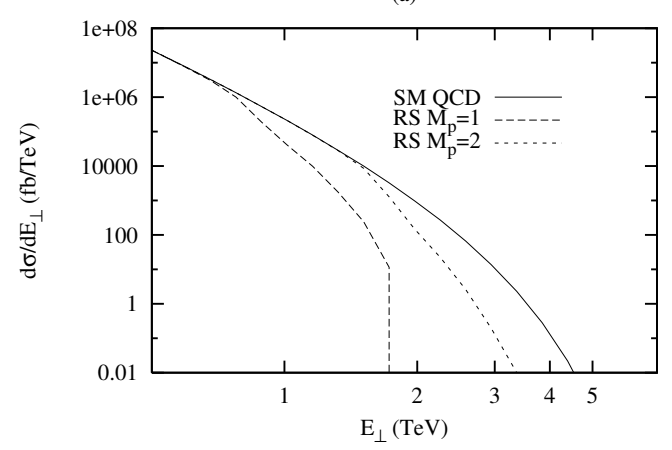

(b)

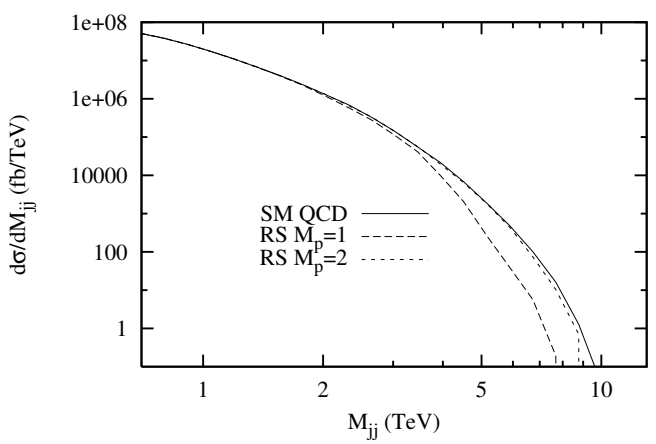

Figure 6: Differential jet cross sections for the RS scenario with $P=1$ and $M_{\mathrm{P}}=1 \mathrm{TeV}$ (longdashed line) and $M_{\mathrm{P}}=2 \mathrm{TeV}$ (short-dashed line), compared with the Standard Model prediction (full line). (a) shows the cross section as a function of the transverse energy of the hardest jet and (b) the cross section as a function of the invariant mass of the two hardest jets in an event.

collider time scales there is no Hawking radiation to camouflage the QCD drop and the absence of QCD events may be a key signal.

The point where the extra-dimensional black hole plus standard model cross section falls below the four dimensional standard model cross section is sensitive to the number of extremely energetic quanta emitted by small black holes and this depends on physics in the Planck region. While we cannot exclude (due to the large theoretical uncertainties) that a drop below the standard model QCD-cross section will be observed at the LHC, it is unlikely (at least in this simple form) to be important for the identification of the ADD model. Inventing mores sophisticated methods for distinguishing the black hole jets from other jets may on the other hand be well worth the effort.

Finally we point out that it is, in a sense, unphysical to consider standard model and black hole events without taking gravitational, non black hole, events into account. Surely the gravitational events will play a major role close to the Planck mass. For scatterings with momentum transfer far above the plank mass we expect black hole events to dominate. We intend to further investigate the effect of gravitational interaction in future publications.

\section{References}

[1] N. Arkani-Hamed, S. Dimopoulos, and G. R. Dvali Phys. Lett. B429 (1998) 263-272, hep-ph/9803315.

[2] I. Antoniadis, N. Arkani-Hamed, S. Dimopoulos, and G. R. Dvali Phys. Lett. B436 (1998) 257-263, hep-ph/9804398.

[3] R. Emparan, G. T. Horowitz, and R. C. Myers Phys. Rev. Lett. 85 (2000) 499-502, hep-th/0003118.

[4] S. Dimopoulos and G. Landsberg Phys. Rev. Lett. 87 (2001) 161602, hep-ph/0106295.

[5] S. B. Giddings and S. Thomas Phys. Rev. D65 (2002) 056010, hep-ph/0106219.

[6] P. Kanti Int. J. Mod. Phys. A19 (2004) 4899-4951, hep-ph/0402168.

[7] T. Banks and W. Fischler hep-th/9906038. 
[8] T. Han, J. D. Lykken, and R.-J. Zhang Phys. Rev. D59 (1999) 105006, hep-ph/9811350.

[9] D. Atwood, S. Bar-Shalom, and A. Soni Phys. Rev. D62 (2000) 056008, hep-ph/9911231.

[10] M. A. Doncheski eConf C010630 (2001) P314, hep-ph/0111149.

[11] L. Randall and R. Sundrum Phys. Rev. Lett. 83 (1999) 3370-3373, hep-ph/9905221.

[12] R. C. Myers and M. J. Perry Ann. Phys. 172 (1986) 304.

[13] D. Karasik, C. Sahabandu, P. Suranyi, and L. C. R. Wijewardhana Phys. Rev. D69 (2004) 064022, gr-qc/0309076.

[14] D. Karasik, C. Sahabandu, P. Suranyi, and L. C. R. Wijewardhana Phys. Rev. D70 (2004) 064007, gr-qc/0404015.

[15] R. Casadio and B. Harms Int. J. Mod. Phys. A17 (2002) 4635-4646, hep-th/0110255.

[16] L. A. Anchordoqui, H. Goldberg, and A. D. Shapere Phys. Rev. D66 (2002) 024033, hep-ph/0204228.

[17] T. Sjöstrand, and others Comput. Phys. Commun. 135 (2001) 238-259, arXiv:hep-ph/0010017.

[18] C. M. Harris, P. Richardson, and B. R. Webber JHEP 08 (2003) 033, hep-ph/0307305.

[19] C. M. Harris and P. Kanti JHEP 10 (2003) 014, hep-ph/0309054.

[20] D. Stump et al. JHEP 10 (2003) 046, hep-ph/0303013.

[21] H. Yoshino and Y. Nambu Phys. Rev. D67 (2003) 024009, gr-qc/0209003.

[22] H. Yoshino and V. S. Rychkov hep-th/0503171.

[23] S. B. Giddings and V. S. Rychkov Phys. Rev. D70 (2004) 104026, hep-th/0409131.

[24] V. S. Rychkov hep-th/0410041.

[25] V. Cardoso, E. Berti, and M. Cavaglia hep-ph/0505125.

[26] C. M. Harris et al. hep-ph/0411022.

[27] D. N. Page Phys. Rev. D14 (1976) 3260-3273.

[28] V. P. Frolov and D. Stojkovic Phys. Rev. D67 (2003) 084004, gr-qc/0211055.

[29] V. P. Frolov and D. Stojkovic Phys. Rev. D68 (2003) 064011, gr-qc/0301016.

[30] D. Stojkovic Phys. Rev. Lett. 94 (2005) 011603, hep-ph/0409124.

[31] V. P. Frolov and D. Stojkovic Phys. Rev. D66 (2002) 084002, hep-th/0206046.

[32] V. P. Frolov and D. Stojkovic Phys. Rev. Lett 89 (2002) 151302, hep-th/0208102.

[33] D. N. Page Phys. Rev. D16 (1977) 2402-2411.

[34] L. Anchordoqui and H. Goldberg Phys. Rev. D67 (2003) 064010, hep-ph/0209337.

[35] CTEQ Collaboration, H. L. Lai et al. Eur. Phys. J. C12 (2000) 375-392, hep-ph/9903282. 